\title{
MÉTODOS DE EVALUACIÓN EN UN CURSO DE ESTADÍSTICA
}

\author{
Ernesto A. ROSA \\ Director del Departamento de Metodología, Estadística y Matemática \\ Universidad Nacional de Tres de Febrero (UNTREF) - República Argentina \\ ear@fibertel.com.ar
}

\begin{abstract}
La Estadística no es una disciplina convencional. En sus contenidos se entremezclan aspectos matemáticos, nociones filosóficas, y fuertes contenidos conceptuales, cuya combinación no se encuentra es otras ciencias. Tampoco lo es su enseñanza o transmisión, ya que dependiendo de la formación básica de los docentes y de su capacidad didáctica, puede llegar a ser una materia incomprensible, o una asignatura entretenida que se convierte en herramienta de utilidad para su aplicación en innumerables disciplinas. La evaluación de los conocimientos que se transmiten en Estadística, tiene aún un mayor nivel de complejidad, ya que se deben considerar: los contenidos del Programa, el enfoque adoptado, la profundidad con que se desarrollan sus técnicas, la disciplina en que se enmarcan y los objetivos que se plantean para la materia dentro de la Carrera. En este trabajo se analizan estas formas de evaluación de los conocimientos transmitidos.
\end{abstract}

\section{PRESENTACIÓN}

El presente texto, corresponde al Trabajo Final del "Seminario de Evaluación de los Aprendizajes Universitarios" realizado en la Universidad Nacional de Tres de Febrero (UNTREF), dentro de un posgrado de Especialización en Docencia Universitaria. Durante el desarrollo del Seminario, se utilizó una nutrida Bibliografía referida al tema de Evaluación, que si bien no ha podido ser incluida totalmente en este texto por problemas de espacio, puede ser solicitada por correo electrónico.

Para el trabajo se optó por la exposición de una "Experiencia en Evaluación" relatada en primera persona, que en realidad se refiere a la evolución de "Las Formas de Evaluación en Estadística", desde el inicio en mis actividades docentes hasta la actualidad.

Naturalmente, para llegar a las "formas de evaluar", debo hacer antes algunas referencias a las "formas de enseñar", aspecto que también se despliega desde mis experiencias iniciales hasta mi desempeño actual en la Carrera de Estadística y en la Maestría en Estadística de la UNTREF.

\section{GÉNESIS DEL TEMA}

Todo tiene un origen. El origen de este trabajo se encuentra mucho antes de mi inicio como docente de Estadística, y hasta antes de saber que esa sería mi disciplina de desempeño profesional y mi tema de enseñanza.

Probablemente el germen del tema se encuentre en la época en que era evaluado en los niveles de enseñanza primaria y secundaria, pero sin dudas la marca más profunda, y que determinó realmente el origen de este trabajo, fue dejada por los exámenes que tuve que rendir en la carrera universitaria que elegí seguir: la Licenciatura en Estadística de la actual Universidad Nacional de Rosario.

Naturalmente, la elección de una carrera de ese tipo, indicaba que no tenía especial antipatía por las Matemáticas, sino todo lo contrario. También era lógico, que esa predisposición hacia los números era compartida por los docentes de las diversas asignaturas del Plan de Estudios, como así también por la gran mayoría de mis compañeros de carrera.

Siendo así, ¿ porqué nos resultaba tan molesto que periódicamente nos evaluaran los conocimientos adquiridos en las materias que cursábamos ? Y no era la inquietud o nerviosismo natural que nos invade ante cualquier examen o situación incierta, sino que era una molestia particular, semejante al efecto de que "nos clavaban una astilla del mismo palo" al que pertenecíamos.

Con los integrantes del grupo de estudio que conformaba, llegamos a la conclusión de que esa sensación de incomodidad o desagrado, no provenía de ser evaluados, sino de la forma o esquema utilizado para la evaluación, y que nos hacía llegar a la situación de tener que escoger (por lo menos para ese momento de la carrera), entre estudiar para aprender o estudiar para aprobar, opción que es uno de los temas centrales en la problemática de Evaluación para los educandos y educadores.

Debido a nuestra predisposición hacia los números, gozábamos de la inspiración de nuestros predecesores en el campo estadístico de hace años y / o siglos, los que partiendo prácticamente de la

In: M.A. Sorto (Ed.), Advances in statistics education: developments, experiences and assessments. Proceedings of the Satellite conference of the International Association for Statistical Education (IASE), July 2015, Rio de Janeiro, Brazil. 
nada, y recurriendo a sutiles e ingeniosos pasos algebraicos, habían desarrollado teoremas y demostraciones que permitirían la aplicación de técnicas y métodos para la resolución de problemas hasta ese momento (el de ellos) irresueltos.

No obstante, habiendo comprendido la base y progresión de esos desarrollos, y teniéndolos a nuestra disposición en la amplia bibliografía que servía de apoyo a nuestros estudios, i no entendíamos la causa de que tuviéramos que memorizarlos, y posteriormente tener que recitarlos o escribirlos, casi sin cambios, en algunas de las evaluaciones incluidas en la programación de las materias! ¿Qué sentido tenía forzarnos a la realización de esos esfuerzos mentales?

También nos resistíamos a tener que memorizar las fórmulas que usualmente remataban esos desarrollos teóricos, sabiendo que en pocos días pasarían a conformar esa masa de recuerdos fusiformes, integrada por remembranzas de los estudios primarios o secundarios (como ser los Teoremas de Pitágoras o de Tales), con los más recientes y complejos desarrollos del nivel universitario (el Teorema Central del Límite, la partición del Chi Cuadrado, la Desigualdad de Tchebycheff, etc.).

Junto con los demás estudiantes del grupo de estudio que integraba, decidimos balancear nuestra forma de trabajo para la incorporación y fijación de los conocimientos que íbamos adquiriendo: sin abandonar la idea primordial de "estudiar para aprender", cada tanto, y en función de las exigencias de los profesores de algunas asignaturas, nos dedicamos a "estudiar para aprobar". De no hacerlo, hubiese peligrado nuestra pretensión de ser profesionales en Estadística.

\section{PRIMERAS EXPERIENCIAS EN DOCENCIA Y EVALUACIÓN}

Poco después de mi graduación comencé a trabajar en una entidad pública en la que me tocó participar de un Curso de Capacitación en Desarrollo Económico Regional, teniendo a mi cargo dos cursos: uno de elementos de Matemática básica y otro de Estadística.

Cabe mencionar que en ninguno de mis niveles de estudio, había recibido alguna capacitación en didáctica o pedagogía, y la única alternativa que tenía era imitar lo que había recibido, intentando replicar lo que consideraba bueno, y mejorar o por lo menos corregir aquellos aspectos que creía negativos. Preparé hasta el cansancio mis clases, intentando ser lo más claro y práctico posible, y preguntando con bastante asiduidad a los cursantes si había algo que no comprendían.

Por suerte (para mi y para los cursantes), la evaluación que se debía tomar no era "eliminatoria", sino que se tenía el objetivo de medir los conocimientos que los cursantes habían captado con el fin de corregir los errores de interpretación y recapacitarlos. La forma de evaluarlos fue de tipo convencional (examen presencial con preguntas conceptuales y aplicaciones prácticas). Lo único "avanzado" para esa época fue que, para sorpresa de los alumnos (y docentes de otras asignaturas del mismo curso), les proporcioné, escritas en la pizarra, las fórmulas que podían aplicar (algo que a mi me hubiese gustado que me hicieran cuando yo estudiaba).

Independientemente de que ese examen no eliminaba a nadie, la sensación de haberles hecho pasar a los cursantes los mismos o similares malos momentos que yo había experimentado, me persiguió durante un tiempo.

Meses después comencé a realizar actividades docentes convencionales en temas de Estadística, actuando como ayudante y / o adjunto en materias de diversas carreras universitarias, donde, en las evaluaciones, mi participación era secundaria, ya que la responsabilidad la tenían los profesores titulares de cada una de ellas. Si bien esa circunstancia me quitaba el peso de tener que decidir la forma de evaluar, no disminuía la molestia de ver sufrir a los alumnos que tenían que rendir exámenes parciales y finales, situación que se acrecentaba en los casos en que debía participar en instancias de exámenes orales.

Debe tenerse presente que hasta ese momento, la única carrera de Estadística existente era la de la Universidad Nacional de Rosario (por lo menos en universidades públicas), y que toda la enseñanza de esa disciplina en el ámbito de la ciudad de Buenos Aires y sus cercanías, se debía desarrollar en carreras de otras disciplinas (ciencias económicas, sociales, biomédicas, exactas, etc.). Ya por ese entonces, a pesar de que el desarrollo de la informática era muy incipiente, la mayoría de las carreras universitarias, incluían en sus planes de estudios materias dedicadas a los métodos estadísticos, obtención y procesamiento de información, etc.

Esta masificación de la inserción de la Estadística en los planes de estudios de la mayoría de las carreras universitarias, se debía a que "esta disciplina es una de las pocas que se entrecruza con 
las restantes y se convierte en indispensable para la descripción y realización de inferencias en la economía, la medicina, la industria, el sector agropecuario, la demografia, la educación, otras ciencias sociales, y cualquier otro aspecto o disciplina que interese abordar" (del "Programa de Enseñanza de la Estadística y Ciencias Afines - PEECA", que está vigente en la UNTREF desde el año 2003, bajo la dirección del autor).

Antes de pasar a otra instancia de la experiencia realizada en la forma de evaluar la capacitación en Estadística, parece oportuno resumir los factores negativos de la Enseñanza de la Estadística que yo había detectado (y sufrido), primero a lo largo de mi época de estudiante, y posteriormente en mi desempeño como colaborador docente.

Básicamente, esos factores negativos, eran las siguientes actitudes de los docentes (naturalmente no todos los docentes presentaban esas falencias):

- Desarrollo de las clases basadas en exposiciones magistrales con nula o escasa participación de los alumnos.

- Equiparación de la Estadística como una parte de la Matemática aplicada.

- Menosprecio por las dificultades de comprensión de los alumnos ("esto no está a su alcance" o "ya lo van a entender cuando avancen en la carrera").

- Desconocimiento o dificultades en la exposición, de las posibles aplicaciones o utilidades posteriores de los desarrollos o métodos expuestos.

- Exposición de ejemplos basados en valores ficticios o sin posibilidades de adaptación a casos reales ("sean los siguientes datos: .......calcular ....").

- Exigencia para las evaluaciones de un manejo fluido y memorizado de largos desarrollos matemáticos, en los que basan su existencia las técnicas que se aplicaban.

- Pretensión, también para las evaluaciones, de la memorización de fórmulas matemáticas.

\section{LA CARRERA DOCENTE EN “LAS LIGAS MAYORES”}

Mi carrera docente se desarrolló dentro de lo que pueden considerarse "causes normales": designaciones en cargos Interinos; concursos para cargos Regulares de Ayudante, Adjunto, y posteriormente de Asociado o Titular; dictado de cursos de Estadística para graduados en otras disciplinas; etc., ganando paulatinamente responsabilidades en el desarrollo de las materias, y por ende en la determinación de los temas y las formas de evaluarlas.

Pero antes de avanzar en esta instancia de mi carrera docente, deben aclararse algunas peculiaridades de la enseñanza de la Estadística.

\section{Las Particularidades de Enseñar Estadística}

En el mismo PEECA mencionado en una aclaración anterior, básicamente en carreras de ciencias económicas, ciencias sociales y posteriormente ingenierías, se plantea como fundamento para la creación del Programa, que "el aprendizaje de las Matemáticas y de todas las ciencias que involucran el uso de números y de las herramientas y operadores que se aplican a ellos, generan entre los estudiantes atracción (en unos pocos) o rechazo (en la mayoría), de acuerdo a la propensión o dificultades que se tengan para comprenderlos y avanzar en sus desarrollos y aplicaciones; y que esta afirmación puede ser aplicada en toda su magnitud al aprendizaje de la Estadística, tema al cual la UNTREF, desde su creación, ha dedicado especial atención en las múltiples facetas que involucran su desarrollo: las actividades docentes de grado y posgrado universitario, la investigación, la difusión, el asesoramiento y consultoría, etc.".

Lamentablemente, cuando comencé a tener la responsabilidad total en el desarrollo de materias de Estadística en carreras de diversas disciplinas (desde mediados / fines de la década del 70'), no existía ese Programa, y eran muy incipientes las investigaciones en otros países sobre aspectos vinculados a la Enseñanza de esa disciplina. Mi único acercamiento al tema, habían sido mis sensaciones como estudiante, y mis primeras experiencias como docente de apoyo.

A esto se agregaba que debido a la escasa existencia de profesionales en Estadística dedicados a la docencia, la mayoría de las asignaturas de esa disciplina existentes en las carreras terciarias o universitarias, eran desarrolladas por matemáticos, o por los graduados en las mismas carreras en que se dictaban esas materias, los que usualmente transmitían a sus alumnos los mismos conocimientos (i y frustraciones ?), que ellos habían recibido. 
Con estas limitaciones y condiciones previas, en mis cursos de Estadística dados en carreras de otras disciplinas, inicié paulatinamente un cambio en la forma de desarrollarlos, basado en los siguientes criterios:

$1^{\circ}$. Presentación de cada tema a partir del planteo de un problema real que debía ser resuelto con métodos estadísticos, resolviéndolo a medida que se presentaban las técnicas estadísticas.

$2^{\circ}$. Omisión de los desarrollos matemáticos innecesarios y de aquellos aspectos vinculados con los cimientos teóricos del pasado, haciendo básicamente hincapié en los aspectos conceptuales.

$3^{\circ}$. Resaltar especialmente las bondades, defectos y limitaciones de los métodos estadísticos expuestos.

$4^{\circ}$. Resumir periódicamente a lo largo del curso, las expresiones matemáticas (fórmulas) derivadas de las técnicas desarrolladas, explicitando sus aplicaciones.

Remarcando lo mencionado en este punto, en relación a la inexistencia de investigaciones sobre estos temas, promediando los $90^{\prime}$, en los congresos y otros eventos de Estadística todavía no existía el rubro "Enseñanza", eran muy escasas las posibilidades de debatir sobre el asunto, y hasta diría que los que sospechábamos que algo se estaba haciendo mal en el tema, teníamos cierta vergüenza de expresarlo públicamente. Temíamos expresiones tales como: ¿Quiénes son estos que vienen a criticar la forma en que se enseña Estadística desde siempre ....?

\section{La Responsabilidad de Evaluar}

Como ya se mencionó en el punto previo, con más de 10 años de ejercer la profesión y ascender en la carrera docente, me llegó el momento de tener la responsabilidad total para decidir sobre el contenido de los Programas de las materias, el enfoque que debería darse a las mismas y, particularmente, sobre el esquema que deberían tener las evaluaciones, situación que me hizo replantear todo el sistema de medición de la captación por parte de los alumnos, de los conocimientos por mi transmitidos (o por mis adjuntos y ayudantes), ya que tuve que definir tanto las formas de evaluación, como los temas a incluir en las mismas, las condiciones previas al momento de realizarlas, las características particulares del mismo momento, y la forma de devolver a los alumnos los resultados que habían logrado.

En esta instancia, y sin tener totalmente claras las ideas sobre las formas de evaluar correctamente la adquisición de conocimientos en Estadística, debía arriesgarme a modificar por lo menos aquellos elementos que más me habian molestado durante mis épocas de estudiante y colaborador docente, los que eran:

- Exigir el recitado automático (oral o escrito) de los desarrollos teóricos, que si bien yo podía o no habérselos desarrollado durante las clases, podían estudiarlos de la bibliografía existente.

- Exigir la memorización de las fórmulas que habían sido presentadas durante las clases y utilizadas para la resolución de casos. La idea era que pudieran ser consultadas durante las evaluaciones.

Quedaría para más adelante el intento de resolver la cuestión de mayor relevancia: lograr que los alumnos estudiaran para aprender y no solo para aprobar (lo que yo suponía que hacía la mayoría).

Las modificaciones aplicadas a las evaluaciones, sorprendieron a todos los alumnos: gratamente a la mayoría y con cierto disgusto a los más resistentes a los cambios; pero sin dudas produjeron modificaciones sustanciales en la forma de estudiar esas materias, con mayor dedicación a los aspectos conceptuales y a las ventajas y limitaciones en la aplicación de las diversas técnicas, y menor tiempo (o ninguno), dedicado a la memorización de "cosas".

Los cambios generados en esos cursos, motivaron una modificación simétrica en el "clima" en que se desarrollaban otros similares de las mismas universidades / facultades, y aunque ello no implicara todavía, un cambio sustancial del enfoque utilizado para el desarrollo de las clases, fueron imitados paulatinamente por docentes de algunos otros cursos.

\section{Otra vuelta de tuerca}

El paso siguiente consistió en intentar reconstruir en el ambiente del aula, la situación que deberían pasar los alumnos que tuviesen que trabajar en temas en los que se requiriera el uso de técnicas estadísticas. Recordemos que eran alumnos de carreras en las cuales la Estadística es una disciplina complementaria (economistas, contadores, ingenieros, sociólogos, etc.), y que se suponía que en algún momento de su actividad profesional, podrían recurrir al uso de algunas de las técnicas 
en ella desarrollada. La enumeración de algunas características imaginadas en ese ambiente laboral al tener que utilizar alguna técnica estadística, es la siguiente:

- Poder consultar cualquier bibliografía que se tenga al alcance. Esto implica y resalta lo innecesario de la memorización de fórmulas y fundamentos.

- Poder recurrir a ejemplos o consulta de experiencias o casos similares al que se enfrenta.

- Tener que resolver el problema "para ayer" (es decir con un tiempo limitadísimo).

- Para la resolución de esos problemas, no se requiere de ninguna manera demostrar que se manejan artilugios matemáticos para fundamentar los instrumentos que se utilizan.

- Poder aclarar con quien encarga el trabajo cualquier duda que surgiera.

- Poder debatir el problema con otros profesionales en un grupo interdisciplinario.

Estas situaciones se practicaban durantes las clases sin ningún tipo de tropiezos, particularmente al realizarse trabajos grupales, durante los cuales cada integrante del grupo podía (o no), jugar un rol diferente.

Las más dificultosas de reproducir en las evaluaciones individuales, eran las dos últimas: aclarar dudas con quien encarga el trabajo, y poder realizar interconsultas con sus compañeros.

El agregado de muchas de estas particularidades a los dos puntos enumerados anteriormente (no incluir desarrollos matemáticos ni memorización de fórmulas), generó una forma de evaluación basada en temarios preparados con los siguientes criterios:

a) Examen "a libro abierto", es decir que los alumnos podían consultar lo que quisieran: libros, apuntes, ejemplos anteriores, "machetes". La preparación de este tipo de instrumentos, era promovida por mi, ya que la preparación del "machete", es una forma excelente de estudiar y resumir los puntos principales del estudio, y dado que durante los exámenes se podía consultar cualquier cosa ..... era ilógico no promover los "machetes".

b) Inclusión de preguntas conceptuales dirigidas a la medición del conocimiento de las nociones básicas de los instrumentos estadísticos, y los aspectos esenciales para su utilización.

c) Inclusión de problemas de resolución práctica, intentando con ellos reproducir las situaciones que se deben enfrentar en la realidad, en las que se deben enfrentar asuntos concretos (simplificados para posibilitar su resolución en los tiempos de un curso o evaluación).

d) Omisión total de las demostraciones que fundamentan los métodos que se utilizan.

e) Aplicación de tiempos estrictos para la resolución del temario, basado esto en querer asimilar en la evaluación, las limitaciones de tiempo que se tienen en la realidad.

f) Una posibilidad no brindada, era la de interconsulta con compañeros. La excusa para esto era que en la realidad, la resolución grupal se realiza con profesionales de otras disciplinas, y los alumnos del curso tenían todos la misma formación.

g) Para evitar (o disminuir), las posibilidades de "interconsulta" entre los alumnos (copiarse), y dado el bullicio natural de las consultas a sus materiales de estudio y la posibilidad de aclarar dudas de interpretación con el docente, los exámenes constaban de gran cantidad de temas diferentes (dependiendo del tamaño del curso, no menos de 6 temas), lo que alejaba la posibilidad de "contactos" debido a las cercanías. Esto, ligado al tiempo escaso que se disponía para responder las preguntas, lograba generalmente que cada uno se dedicara a "su" tema, sin preocuparse por los de los demás.

h) Restricciones en la posibilidad de aclarar dudas con quien encargaba el trabajo (el docente), aunque como hacer esto les limitaba el uso del tiempo disponible para responder las preguntas, era administrado con sobriedad por los mismos alumnos.

Este esquema de trabajo (tanto en el enfoque para el desarrollo de las clases, como por las formas de evaluar los conocimientos adquiridos), era medido periódicamente con encuestas realizadas a los alumnos por cada una de las casas de estudio, o en su defecto por las cátedras que yo coordinaba, resultando con opiniones muy favorables, logrando por lo menos que el concepto y visualización de la Estadística no fuera tan negativo, y que se la despegara parcialmente de la Matemática pura.

Naturalmente, lo más criticado por los alumnos en esas encuestas, era el "escaso tiempo disponible para responder los exámenes", lo que estaba muy correlacionado con aquellos alumnos que habían dedicado poco tiempo previo al estudio, confiando (pese a todas mis advertencias), en que la situación de "libro abierto", les permitiría compensar durante el examen lo no realizado previamente.

CONCLUSIONES 
Intentando sintetizar los diversos aspectos y argumentos expuestos en los puntos anteriores, puede decirse que:

1. No es posible evaluar de la misma forma los conocimientos en Estadística que se transmiten en Carreras específicas del tema, que aquellos que se brindan como conocimientos complementarios en carreras de otras disciplinas.

2. En las asignaturas de las carreras de Estadística, es aceptable que en las evaluaciones se exija el conocimiento de algunos desarrollos teóricos básicos en determinados temas (los conceptos y las argucias matemáticas a las que se recurre), siendo esto innecesario e inconducente en las materias de Estadística de las carreras de otras disciplinas.

3. En ningún caso es aceptable que se exija a los alumnos la memorización de las innumerables fórmulas que contienen los métodos estadísticos, sugiriéndose la conveniencia de ir preparando durante el curso uno o varios resúmenes de las expresiones algebraicas necesarias.

4. Las evaluaciones deben cubrir básicamente el conocimiento de los alumnos de las aplicaciones de las diversas técnicas desarrolladas, las particularidades o condiciones para hacerlo, las bondades de sus usos y las limitaciones de cada una.

5. Los aspectos conceptuales pueden ser evaluados mediante la técnica de selección ante alternativas múltiples, mediante las cuales pueda graduarse el grado de conocimiento de los alumnos sobre las particularidades y posibilidades de aplicación de los métodos estadísticos.

6. Conveniencia de la utilización de la técnica de evaluación "a libro abierto" (es decir que los alumnos puedan consultar el material que quieran durante el momento del examen).

7. Como contrapartida se debería aplicar un tiempo bastante estricto y limitado para finalizar la evaluación. De no hacerlo así, teóricamente cualquier alumno "sagaz" teniendo tiempo ilimitado, sin haber estudiado previamente, podría responder sin inconvenientes cualquier cuestionario.

8. El argumento para aplicar estas dos sugerencias, es que se reproduce lo que usualmente se tiene en la realidad laboral al enfrentarse la aplicación de técnicas estadísticas.

\section{BIBLIOGRAFÍA}

* Aburto Luyo, Huber (2000). "Evaluación de los Aprendizajes". Min. Educación Perú.

* Alvarez Mendez, J. M. (2001). "Evaluar para Conocer, Examinar para Excluir", Madrid: Morata.

* Carballo, R. (1990). "Evolución del Concepto de Evaluación. España.

* Carballo, R. (2000). "El Análisis de Datos en la Evaluación de Programas Educativos". Revista de Investigación Educativa, Vol. 18, n. ${ }^{\circ}$ 2, págs. 357-379, España.

* Casanova, María A. (1995). "Manual de Evaluación Educativa”. Ed. Muralla. España.

* DINIECE - "La Evaluación de la Calidad Educativa en Argentina: Experiencias Provinciales". Min. Educación. R. Argentina.

* García Martínez, F. A. (2005). "La Evaluación en el Complejo Mundo de la Educación”. Etic@net, Año II, No 5. Granada, España.

* Garcia Ramos, J.M. (1989) - "Bases Pedagógicas de la Evaluación: Síntesis". DialNet. Madrid. España.

* Lafi, Paola (2003). "La Paradoja de la Evaluación Educativa: Evaluar para Aprobar vs. Evaluar para Aprender". Mendoza, R. Argentina.

* Litwin, E. (1998). "La evaluación de los Aprendizajes en el Debate Didáctico Contemporáneo". Ed. Piados Educador. Buenos Aires, R. Argentina.

* OCDE (1995) - "Measuring What Students Learn".

* Osoro J.M.(1995) - "Los Indicadores de Rendimiento en la Evaluación Institucional Universitaria" - ICE - Universidad de Zaragoza. España.

* Popham, W.J.(2008) - "La Evaluación Referida a Criterios". Cultura Evaluativa. México.

* Santos Guerra, Miguel. A. (1999). "20 Paradojas de la Evaluación del Alumnado en la Universidad Española”. Revista Electrónica Interuniversitaria. Univ. de Granada. España.

* Fundación Instituto Ciencias del Hombre (2005). "La Evaluación Educativa: Conceptos, Funciones y Tipos". Madrid. España.

* Vázquez Alonso, Ángel (2001). “La Evaluación del Sistema Educativo”. Rev. Educación y Cultura (247 / 274). Islas Baleares, España.

* www.evaluacion.edusanluis.com.ar. "La Evaluación Educativa como Derecho Humano". Univ. Nac. San Luis. R. Argentina. 